\title{
Surface analysis and roughness parameters of Copper Metallic
}

\author{
Halo Dalshad Omar ${ }^{1}$
}

\author{
${ }^{1}$ Department of Physics, Faculty of Science and Health, Koya University \\ Daniel Mitterrand Boulevard, Koya KOY45 AB64, Kurdistan Region - Iraq
}

Email address: I: halo.dalshad@koyauniversity.org

\section{Keywords: Topography, STM, Roughness parameter, XRF, XRF analysis}

\begin{abstract}
The level of copper $(\mathrm{Cu})$ distribution in the hard alloy depends little on powder material hardness although the other mechanical properties of the materials and the crystal structure of copper is face centered cubic (FCC). A discussion about simple and low cost preparation of copper powder by (Mini Mill 2 Panalytical) and preparation of the sample was rotating at 5 min and in the case of grinding of samples at high speed $300 \mathrm{rpm}$. The surface morphology of copper metallic powder was determined with Scanning Tunneling Microscope (STM) (Model: NTMDT Solver Nano.). The characterization of surface topographyand surface measurement determines surface topography and surface measurement copper includes roughness parameter. X-ray fluorescence analysis is a powerful analytical tool for the determination of almost all the elements present in a sample. The spectra obtained were analyzed using a X-ray fluorescence (XRF) (Model: Rigaku-NEX CG). Each element emits a unique spectrum of X-rays characteristic of that element. From the spectra obtained, there was the element to be present in the sample was copper $(\mathrm{Cu})$.
\end{abstract}

\section{INTRODUCTION}

The copper atom is quite similar to an atom of gold or silver [12]. Within the copper atom lattice a cloud of free electrons is uniquely available for the transfer of electrical current. Solid copper can be described as the arrangement of copper atoms in a face-centered-cubic configuration [14]. A copper atom is found at each corner and in the center of each face of a cube [10-19]. This is the unit cell which is repeated in three dimensional space to make up the crystal structure of the metal [16-18]. Scanning Tunneling Microscope (STM) in 1982 at IBM in Zurich by Binning. The tip-sample interaction in STM is based on a tunneling electrical current [15]. Although the ability of the STM to image and measure the material surface with atomic resolution [20]. To better understand the morphology of a surface a quantitative description of the surface topography must be carried out. The topography matrix data should be treated in each profile line (2D). The amplitude parameters are the principal parameters in characterizing the surface topography. The root mean square roughness $(\mathrm{Rq})$ are the most used amplitude parameters. The reason for thisis that this parameter is more sensitive to large deviations with respect to the mean line. To know if the $\mathrm{Rq}$ values are making sense, the height points have to be uniformly distributed according to a normal distribution curve. The radiation intensity of each element signal, which is proportional to the concentration of the element in the sample [5]. This excitation ejects electrons from the atomic shells of the elements in the sample. When a given atom replaces the ejected electron, by taking another electron from an outer atomic shell, x-ray energies are emitted [6]. Since each element generates a specific energy level in this replacement process, these energies are known as characteristic X-rays [2]. The binding energy of the characteristic X-ray lines depend only on the atomic energy levels so are always the same [3]. The measured intensity of an emission lines depends on the number of corresponding atoms in the sample which were excited but on many other parameters aswell. The probability of X-ray emission depends on the element and on the energy of the excitation source [4-9]. The probability of detecting the X-rays depends on the detector sensitivity, attenuation in the sample itself, etc [7]. 


\section{COPPER METTALIC PREPARATION}

Before grinding copper metallic powder via Mini Mill 2 Panalytical was cleaned the bowl with acetone for a short time. The hardness of the grinding bowl used and of the grinding balls must be higher than of the $\mathrm{Cu}$ used [17]. The grinding bowls and grinding balls of copper are resistance to acids with the exception of hydrofluoric acid. In accordance with the application, the grinding time should be adapted of the bowl. Copper metallic powders have investigated at $5 \mathrm{~min}$ and in the case of grinding of samples at high speed $300 \mathrm{rpm}$.

\section{SCANNING TUNNELING MICROSCOPIC STUDY OF COPPER METALLIC}

STM is defined as a specific type of microscopy that uses the basic principle of scanning a surface with a very sharp probe to image and measure properties of material.The STM provides real space atomic resolution images through tunneling current between a tip (Pt-Ir) and an copper surface. The probe is a very important component of a SPM because different probes can measure different properties of the sample. The movement of the tip or sample (copper) in the $x, y$, and zdirections is controlled by a piezoelectric tube scanner, similar to those used in STM. The scanner moves across the first line of the scan, and back. The surface morphology of copper metallic powder was studied by scanning tunneling microscopic as shown in the fig.1. The STM analyses the surface of solid objects, producing images of higher resolution. It produces representation of two dimensional sample from a diverse range of material. From STM images it was found that the root mean square roughness that the image $40 \times 40$ um scanning areas with $2000 \times 2000$ points, and scanning rate of $3.286 \mathrm{~Hz}$, applied voltage $2.53 \mathrm{nVand}$ the current between surface iron metallic and tip was $4.825 \mathrm{nA}$.

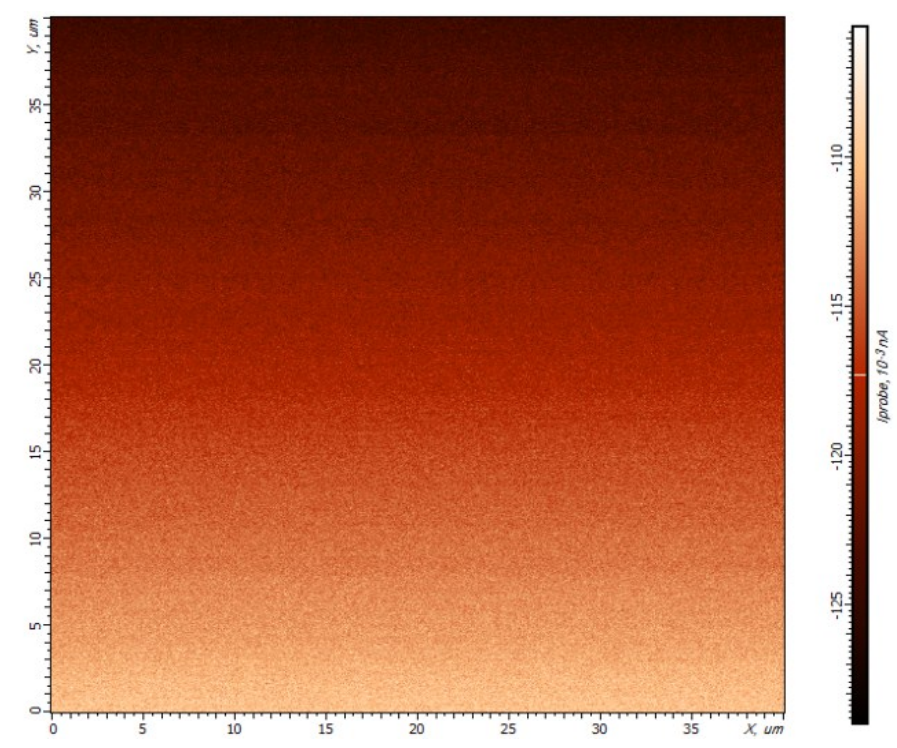

Fig.1. STM image for surface morphology of 2D of copper metallic 40 x $40 \mathrm{um}$.

\section{ROUGHNESS PARAMETER: (PEAKS, VALLEYS AND AVERAGE OF ORDINATES)}

The characterization of surface topographyand its understanding is important in procedures involving friction, greasing and wear [22]. A very important thing to consider is that the variation of the radius of stylus tip may affect the shape of the profiled surface because the radius of the tip of the pen draws a single envelope of the actual profile [24]. 
4.1 Maximum profile valley depth of the roughness profile( $R v)$, Maximum profile peak height of the roughness profile (Rp) and Total height of the roughness profile(RT)

The Maximum Profile Peak Height (Rp) is the measure of the highest peak around the surface profile from the baseline [23]. Likewise the Maximum Profile Valley Depth (Rv) is the measure of the deepest valley across the surface profile analyzed from the baseline and $\mathrm{Z}$ is profile of surface as shown in the fig. (2). We can write:

$$
\begin{aligned}
& R_{p}=\left|\max Z_{(x)}\right| \\
& R_{v}=\left|\min Z_{(x)}\right|
\end{aligned}
$$

Thus the Maximum Height of the Profile (RT) can be defined as the vertical distance between the deepest valley and highest peak.

$$
R_{T}=R_{p}+R_{v}
$$

\subsection{Root mean square deviation of the roughness profile $(\mathrm{Rq})$}

The root mean square (RMS) is a statistical measure used in different fields. We cite, as an example, the use of the RMS amplitude applied to harmonic oscillators, such as on an alternating electric current. The root mean square of roughness $(\mathrm{Rq})$ is a function that takes the square of the measures [21]. The RMS roughness of a surface is similar to the roughness average, with the only difference being the mean squared absolute values of surface roughness profileas shown in the fig. (2). The function $\mathrm{Rq}$ is defined as:

$$
R_{q}=\sqrt{\frac{1}{l} \int_{0}^{l}\left|Z^{3}(x) d x\right|}
$$

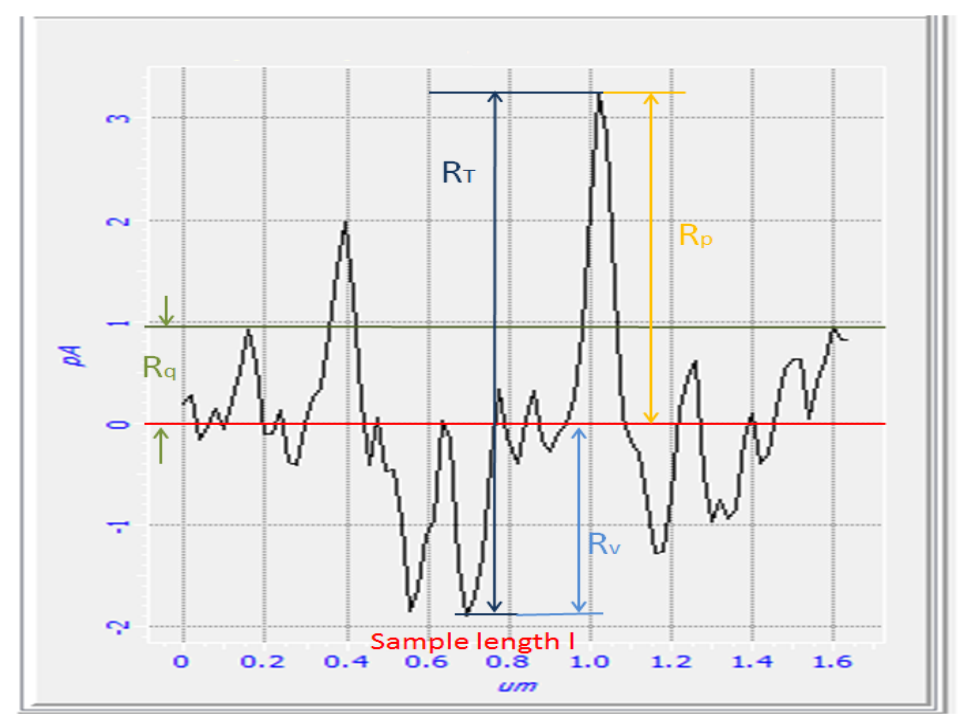

Fig.2. Illustration of Maximum Height of the Profile (RT), Maximum Profile Valley Depth (Rv), Maximum Profile Peak Height (Rp) for the surface profile and Root mean square deviation of the roughness profile $(\mathrm{Rq})$.

\section{X-RAY FLUORESCENCE APPLIED TO COPPER ANALYSIS}

The plot below show how characteristic X-ray spectra are used to determine the element which is present in a sample [8-11]. When the detector absorbs fluorescence, its conductance changes in proportion to the energy of the fluorescence, which then is processed by the electronics [1-2]. The 
intensity, or frequency of occurrence measured in counts per second, on the vertical axis and energy of the fluorescent signal, measured in kiloelectronvolts, across the horizontal axis as shown in the fig. (3).The fluorescent energy identifies the element, while the intensity of the fluorescence is a measure of the element's concentration [13]. The measured intensity of a single emission line, say the $\mathrm{Cu} \mathrm{K} \alpha$ peak above, depends on the energy spectrum and the intensity of the exciting X-rays, on the efficiency of the X-ray detector, and on the geometry of the source and sample. It also depends on the other elements in the sample. The tube voltage and tube current can be set within $25 \mathrm{kV}$ and $1.0 \mathrm{~mA}$ respectively. Copper has a strong peak at $8.047 \mathrm{keV}$ and has a weak at $8.905 \mathrm{keV}$ as shown in the fig.3.

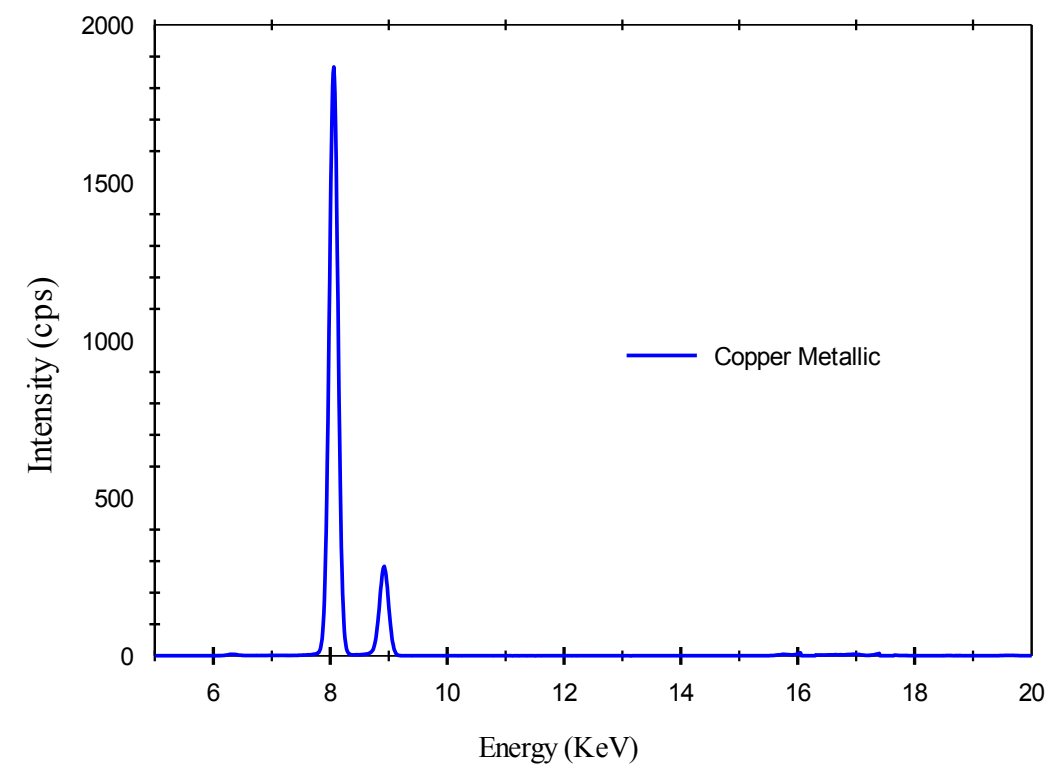

Fig.3. Shows the measured energy spectrum and identifies the major peaks copper.

\section{RESULT AND CONCLUSION}

The surfaces of the products of copper metallic were obviously roughness. Surface measurement determines surface topography, includes roughness as shown in the fig. (1). Similar to some surface properties such as hardness, the value of surface roughness depends on the scale of measurement. In STM, the Rq depends on the swept area of the sample, the scan size. The Rq is more sensitive to peaks and valleys than the average roughness due to the squaring of the amplitude in its calculation. The evaluated maximum profile valley depth of the roughness profile(Rv) surface roughness of the sample was $1.877(\mathrm{pA})$, maximum profile peak height of the roughness profile $(\mathrm{Rp})$ was $3.320(\mathrm{pA})$, total height of the roughness profile(RT) was 5.197(pA) and Root mean square deviation of the roughness profile $(\mathrm{Rq})$ was $0.952(\mathrm{pA})$ for copper sample, as seen in the Fig. (2). The measured intensity of single emission line depends on the energy spectrum and of exciting Xrays, on the efficiency of the detector and the geometry of source and sample. It also depends on the other elements in the sample. The XRF study confirms / indicates that the resultant of the copper metallic powder by the spectrum obtained, there were a strong peak at $8.047 \mathrm{keV}$ and has a weak at $8.905 \mathrm{keV}$ as shown in the fig.3.

\section{Acknowledgements}

Author wishes to thank and supporting of this investigation by department of physics/ Faculty of Science and Health/University of Koya/ Kurdistan Iraq. 


\section{Refrence}

[1] Laura A. Hutton, Glen D. O’Neil, Tania L. Read, ZoëJ. Ayres, Mark E, Electrochemical X-ray Fluorescence Spectroscopy for Trace Heavy Metal Analysis: Enhancing X-ray Fluorescence Detection Capabilities by Four Orders of Magnitude, 05 July 2015.

[2] Kenji SAKURAIand Hiromi EBA, X-Ray Fluorescence Analysis with a Johansson-type Spectrometer, National Research Institute for Metals, 1-2-1, Sengen, Tsukuba, Ibaraki 305-0047, Japan, 26 November 1998.

[3] Selma M. H. Al-Jawad, Determined the parameters effected on sensitivity and lower limit detection of XRF-WDS for different metals particles suspended in engine oil, Determined the parameters effected on sensitivity and lower limit detection of XRF-WDS for different metals particles suspended in engine oil, Journal of Kirkuk University - Scientific Studies, vol.4, No.1,2009.

[4] Clark, D. and Mark, B., Determinin trace element in Water using Microsample X-ray analysis, pp.90-97 (2000).

[5] Eugene,B., Principles and Practice of X-ray Spectrometric Analysis,Second Edition,Plenum Press NewYork (1975).

[6] Ron,J., Chemical analysis - X-ray fluorescence spectrometry Publishers,John Wiley and Sons,NewYork (1988).

[7] Robert, R. w., X -ray methods for monitoring machinery. Proceedings of the 45th Annual Denver X-ray Conference publication in advances in X-ray analysis, Vol.40, (1996).

[8] Kenji SAKURAIand Hiromi EBA, X-Ray Fluorescence Analysis with a Johansson-type Spectrometer, National Research Institute for Metals, 1-2-1, Sengen, Tsukuba, Ibaraki 305-0047, Japan,26 November 1998.

[9] B. D. Cullity and S. R. Stock, Elements of X-Ray Diffraction, Associate Professor of Metallurgy in University of Notre Dame, 1978.

[10] Charles Kittel, Introduction to Solid State Physics(ISBN 047141526X) Wiley, 8th Edition, 2004.

[11] Fairuz M. N, Bauk S. and Suzana M. I., XRF study on some Indian Mackerel samples, IJBAS IJENS Vol: 12 No:03, June 2012.

[12] Viktor Hauk, Nin-destructive methods of measurement of residual stresses, Imtitut fiir, Rhei zisch-Wesfilische Technische Hochschule Aachen, D-5100 Aachen, FRG, advances in surf. Treatments vol.3, 87120 .

[13] Bradner D. Wheeler, analysis of limestones and dolomites by X-ray fluorescence, The Rigaku Journal, Rigaku/USA, 199 Rosewood Drive, Danvers, MA 01923, USA, Vol. 16/ number 1/ 1999.

[14] Alan Lawley, International Journal of Powder Metallurgy - Focus Issue: Precious Metals, Platinum Metals Rev., 54, (2), 122-124, 2010.

[15] T Machleidt, E Sparrer, D Kapusi and K-H Franke, Deconvolution of Kelvin probe force microscopy measurements - methodology and application, measurement science and technology, 20 (2009) 084017 (6pp).

[16] R. Saravanan, M. Prema Rani, Metal and Alloy Bonding - An Experimental Analysis: Charge Density in Metals and Alloys, springer, September 20, 2011.

[17] Halo Dalshad Omar, To Investigation the Structure and Morphology of Iron Metallic by Difference Techniques,Department of Physics, School of Science, University of Koya, J. Nano. Adv. Mat. 3, No. 2, 57-61, 2015. 
[18] I. N. Svinolobova, P. N. Ostrik, and A. N. Kovzik, Properties of iron-copper master alloy powder, Powder Metallurgy and Metal Ceramics, Vol. 36, Nos.11-12,1997.

[19] R.A. Flinn, Copper, brass and bronze Castings, Non-Ferrous Founders' Society, Inc., Cleveland, 1961.

[20] Mohammad Ghaffar Faraj, Effect of Thickness on the Structural and Electrical Properties of Spray Pyrolysed Lead Sulfide Thin Films, American Journal of Condensed Matter Physics 2015, 5(2): 51-55.

[21] M. Raposo, Q. Ferreira andP.A. Ribeiro, A Guide for Atomic Force Microscopy Analysis of SoftCondensed Matter, A. Méndez-Vilas and J. Díaz (Eds.) 2007.

[22] Wilson, R.A., \& Bullen, H. A., Analytical Sciences Digital Library,2007.

[23] Muhammad Azam, Mirza Jahanzaib, Ahmad Wasim, Salman Hussain, Surface roughness modeling using RSM for HSLA steel by coated carbide tools, May 2015, Volume 78, Issue 5-8, pp 1031-1041.

[24] Yoshiharu Namba, Jin Yu, Jean M. Bennett, and Koujun Yamashita, Modeling and measurements of atomic surface roughness, Vol. 39, Issue 16, (2000). 